\title{
Data Marketing Optimization Method Combining Deep Neural Network and Evolutionary Algorithm
}

\author{
Wei Wang (iD \\ Economics and Management Department, Chengdu Normal College, Chengdu 611130, China \\ Correspondence should be addressed to Wei Wang; weiwang@cdnu.edu.cn
}

Received 2 December 2021; Accepted 3 January 2022; Published 31 January 2022

Academic Editor: Deepak Kumar Jain

Copyright (c) 2022 Wei Wang. This is an open access article distributed under the Creative Commons Attribution License, which permits unrestricted use, distribution, and reproduction in any medium, provided the original work is properly cited.

\begin{abstract}
The design and optimization of personalized marketing strategy have become an important direction for e-commerce enterprises to meet the differentiated needs of consumers, innovate service content, and improve their core competitiveness. It is important to analyze the characteristics of personalized marketing in the e-commerce environment and to study and establish the optimization model of personalized marketing strategy and the model solving method, which are suitable for the application environment. In order to develop a new e-commerce model for consumers, innovate the online service content of enterprises, and improve consumer satisfaction, this paper improved two topological weight evolution methods of evolutionary neural networks and used them as tools for model solving, with the objectives of attracting potential consumers, improving consumer satisfaction, and maximizing revenue. The results of the experiments show that the proposed model is a good one. The experimental results show that the optimization model and model solving method proposed in this paper can efficiently build consumer demand and preference models from large-scale data and can help e-commerce enterprises to formulate accurate personalized promotion and pricing strategies to maximize their profits.
\end{abstract}

\section{Introduction}

As a new type of networked economic activity, e-commerce has developed by leaps and bounds in recent years, becoming an important part of the national economy. E-commerce will play a more important role in the national economy [1]. Developing e-commerce and promoting the development of national economy informatization have become an important force to promote China's economic development. The promotion of consumer-oriented e-commerce applications and the development of new consumer-oriented e-commerce models and innovative service content have become one of the guiding directions for the development of e-commerce in China. In the e-commerce environment, consumer needs are becoming more and more differentiated, and the abundance of product choices in the "heterogeneous economy" makes consumer behavior more and more innovative and variable. In this context, analyzing consumers' subjective preferences, adapting to their differentiated needs, and mastering the changing patterns of consumer purchasing behavior have become the core concerns of enterprises, and the design and application of personalized marketing strategies are effective means to solve this problem [2]. With the use of personalized marketing strategy, e-commerce enterprises on the one hand analyze the online behavior of consumers, to provide products and services in line with the individual needs of consumers, and on the other hand, at a lower cost, implement personalized promotion strategy and price strategy, to guide the purchase behavior of consumers. Personalized marketing strategy has become an important direction for e-commerce enterprises to innovate their service content and improve their core competitiveness.

A large number of theoretical studies and application practices have proved that personalized marketing strategy has a positive effect on enterprises to improve consumer satisfaction and loyalty and improve profits and core competitiveness [3-5]. At present, the main research idea of personalized marketing is to design strategies centering on product, promotion, price, and channel $[6,7]$. In the 
e-commerce environment, enterprises provide consumers with personalized products and services through the convenient "marketing channel" of "e-commerce website." Therefore, personalized marketing strategy in e-commerce environment focuses on product strategy, promotion strategy, and price strategy based on enterprise e-commerce website [8]. Figure 1 represents the schematic diagram of personalized marketing in the e-commerce environment.

Through the task analysis of personalized marketing, it can be seen that the design of personalized marketing strategy needs to realize the decision-making tasks at different stages, such as data analysis, scheme synthesis, problem modeling, and model solving [9]. The complicated factors make the modeling and optimization of personalized marketing strategy very difficult. Therefore, the design of personalized marketing strategy needs to make comprehensive use of a variety of intelligent optimization methods to model, solve, and optimize the problem according to the decision-making tasks at different stages [10]. Neural network is an intelligent optimization method for modeling and solving complex problems by simulating human thinking mode [11]. Neural network methods abstract and simulate the basic learning processes of the human brain or natural neural system by constructing complex network systems consisting of a large number of simple neurons that are widely interconnected. Due to its strong robustness, nonlinear mapping ability, strong memory, and self-learning ability, neural network is widely used in large-scale data processing and analysis tasks, such as attribute weight measurement [12], online consumer division [13], web content recommendation [14], etc. The above research models large-scale complex problems and uses the functions of associative learning and self-organization of neural networks to achieve rapid optimization of problems.

It can be said that the research of neural network has made a lot of remarkable achievements, but there are still many shortcomings in the practical application of neural network. Evolutionary algorithm is a heuristic search algorithm [15], its ideas are borrowed from Darwin's evolution and Mendel's genetic theory, and evolutionary algorithm is to simulate the evolution process of biological population in nature, in order to improve their own population's ability to adapt to the environment. In view of the difficulty in constructing the network structure and the difficulty in selecting the super parameters in the neural network, more and more scholars at home and abroad began to study the evolutionary meridian algorithm which combines the evolutionary algorithm with the neural network. The early evolutionary neural networks were fixed network structures and optimized only the weights of the network. For example, Whitley encoded the weight into an 8-byte string [16]. Then, genetic algorithm was used to search for the weight, so as to find the optimal network weight. However, it is far from enough to only optimize the weight; the neural network structure also needs to be built manually, so an excellent evolutionary neural network should also have the ability to choose the optimal neural network structure adaptively. Therefore, Lander proposed a new evolutionary neural network algorithm [17], which can optimize AE as a basic individual unit in the population and generate a more complex neural network structure by combining a large number of such basic individual units. $\mathrm{Ku}$ et al. proposed a coevolution algorithm to optimize timing good and network [18]. They decomposed the evolution of weight and structure into two subproblems and realized the simultaneous evolution of weight and structure on this basis. In recent two years, not only are many outstanding scholars at home and abroad studying evolutionary neural networks, but also many large companies and scientific research institutions, such as OpenAI [19] and Google [20], are further studying the theory and application of evolutionary neural networks. With the further development of neural networks and evolutionary algorithms and the improvement of hardware computing capability, the research on evolutionary neural networks is reviving.

This paper mainly researches and studies the theory of evolutionary neural network, analyzes the advantages and disadvantages of existing evolutionary neural network, then improves the characteristics of evolutionary neural network in some detail, and finally conducts experiments on its application in e-commerce marketing problems.

\section{Modeling Method of Personalized Marketing Strategy}

Existing researches on how to attract consumers to buy recommended products lack effective means. The potential problem is that consumers find products they are interested in through a company's recommendation system but buy them from a competitor's website. Therefore, through corresponding marketing strategies, such as promotion strategy and price strategy, attracting consumers to buy recommended products is of great significance to improve corporate profits and competitiveness [21].

2.1. Assumptions of the Model. Price discount is an effective means for enterprises to attract consumers to buy goods [22]. Although price promotion reduces the profit margin of promotional products, due to the increase in sales of related products, enterprises can often get more income from promotional activities. Therefore, this paper assumes that the original price of the promotional product is the optimal price when the product is sold separately, that is, the price that can bring the best profit for the enterprise without considering the influence of related products.

Substitutes of promotional products refer to products that can meet the same needs of consumers as promotional products, while complementary products refer to products that need to cooperate with promotional products to meet specific needs of consumers. In this paper, complementary, substitute, and independent products of promotional products are distinguished according to product functions.

Personalized recommendation can not only help consumers find the products they are interested in, but also change their purchase intention. In this paper, the above two aspects of personalized recommendation are modeled to obtain products that consumers are interested in by product 


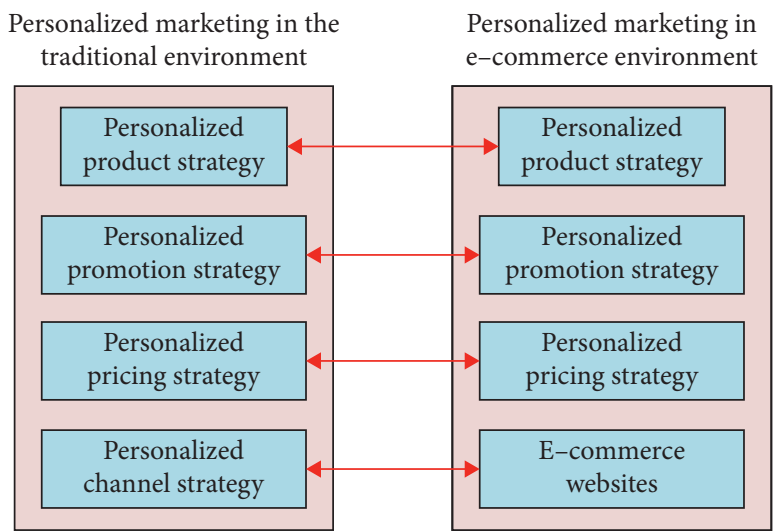

FIGURE 1: Schematic diagram of personalized marketing in the e-commerce environment.

recommendation, and the influence of personalized recommendation on purchase intention is abstracted by the change of consumer reserve price.

2.2. Parameters of the Model. Assume that the current price, cost, and consumer reserve price for product $g$ are $p, c$, and $R_{g}$, respectively. "Consumers have different reserve prices for different products" has been widely recognized in the field of marketing $[23,24]$. Reserve price is a comprehensive evaluation of product value by consumers according to current demand and is an important factor to decide whether to buy. On the premise that enterprise credit, payment, and payment security are guaranteed, (consumers' reserve price for products - actual sales price of products) constitutes consumers' residual utility. Only when the residual utility is greater than 0 , consumers are likely to take the purchase behavior.

Substitute, complementary, and independent products of product $g$ are expressed as SUB $=\left\{g_{1}^{S}, \ldots, g_{s}^{S}, \ldots, g_{\mid \text {SUB }}^{S}\right\}$, $\mathrm{COM}=\left\{g_{1}^{S}, \ldots, g_{s}^{S}, \ldots, g_{|\mathrm{SUB}|}^{S}\right\}, \quad$ and IND $=\left\{g_{1}^{I}, \ldots, g_{i}^{I}, \ldots, g_{|N D|}^{I}\right\}$. The selling prices are expressed as $\quad\left\{c_{1}^{s}, \ldots, c_{c}^{s}, \ldots, c^{C}|\mathrm{SUB}|\right\}$, $\left\{c_{1}^{C}, \ldots, c_{c}^{C}, \ldots, c^{C}|\mathrm{COM}|\right\}$, and $\left\{c_{1}^{I}, \ldots, c_{c}^{I}, \ldots, c^{I}|\mathrm{IND}|\right\}$. It is assumed that there are $M$ potential consumers in the market whose goal is to maximize surplus utility, and they are willing to maximize utility by choosing different products in promotion activities. Consumers will buy a product only when the residual utility of a product is nonnegative and greater than the residual utility of related substitutes. Consumers' reserve prices for substitutes, complementary products, and independent products are $\left\{R_{1}^{S}, \ldots, R_{s}^{S}, \ldots, R_{|\mathrm{SUB}|}^{S}\right\}, \quad\left\{R_{1}^{C}, \ldots, R_{s}^{C}, \ldots, R_{|\mathrm{COM}|}^{C}\right\}, \quad$ and $\left\{R_{1}^{I}, \ldots, R_{s}^{I}, \ldots, R_{|I N D|}^{I}\right\}$. This paper divided consumers into 12 groups based on their presale buying decisions. Figure 2 shows the classification of consumers based on product purchase decisions.

\section{Evolutionary Deep Neural Networks}

Evolutionary neural network is a new algorithm which combines evolutionary algorithm and neural network. In the algorithm of evolutionary neural network, various parameters of neural network will be transformed into chromosomes in evolutionary algorithm through coding operation, and network construction and parameter optimization will be carried out through evolutionary algorithm. As a preliminary attempt to apply the evolutionary algorithm to neural network, the fixed topology method proves the feasibility of applying the evolutionary algorithm to neural network, but at the same time, it still needs to be improved. So weight topology evolution becomes the focus of evolutionary neural network research. However, because of the uncertainty of the network structure, the weighted topology evolution method has many problems that the fixed topology evolution method does not have. Therefore, this paper focuses on the evolutionary neural network based on enhanced topology in the weight topology evolutionary algorithm of neural network and proposes an improved method.

3.1. NEAT Algorithm. Firstly, two elements are needed to construct a complete neural network model: network topology and connection weight. In order to record these two elements completely, NEAT algorithm encodes a complete neural network into two genes: node gene and weight gene. As shown in Figure 3, the weight gene content will be richer, mainly consisting of the following parts: the information of the two nodes connected to the weight, the weight information, whether the connection is available, and the innovation ID.

Compared with the traditional coding method, NEAT can not only restore the structure of neural network well, but also avoid multiple coding methods for the same network. In addition, this kind of coding method provides very favorable support for the operation of paternal gene production and offspring generation, such as mutation operation and crossover operation.

The main methods for NEAT to generate child genes from parent genes are mutation operation and crossover operation. In order to support such operation and avoid structural duplication, NEAT introduces innovation ID to record the history of gene crossover and mutation. Because innovation ID can find the corresponding gene source for each node in the gene, all gene points are derived by 


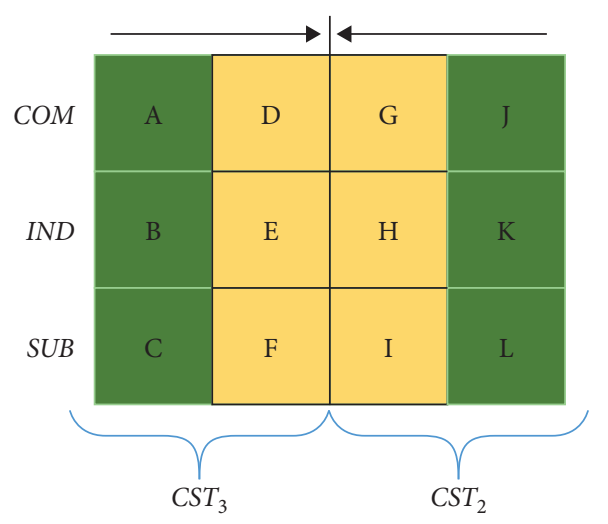

A and J: Consumers who buy COM products before promotion.

$\mathrm{D}$ and $\mathrm{G}$ : consumers who do not buy $C O M$ products before promotion.

$\mathrm{B}$ and K: Consumers who bought an IND product before the promotion.

$\mathrm{E}$ and $\mathrm{H}$ : Consumers who did not buy an IND product before the promotion.

$\mathrm{C}$ and L: consumers who purchase products in SUB before promotion .

$\mathrm{F}$ and I: consumers who do not buy products in $S U B$ before promotion.

$C O M, I N D$ and $S U B$ consumer groups whose needs are not affected by promotions

$C O M, I N D$ and $S U B$ consumer groups whose needs are affected by promotions

FIgURE 2: Classification of consumers based on product purchase decisions.

\begin{tabular}{|c|c|c|c|c|c|}
\hline Node gene & $\begin{array}{c}\text { Node } 1 \\
\text { input }\end{array}$ & $\begin{array}{c}\text { Node } 2 \\
\text { input }\end{array}$ & $\begin{array}{c}\text { Node } 3 \\
\text { input }\end{array}$ & $\begin{array}{c}\text { Node } 4 \\
\text { input }\end{array}$ & $\begin{array}{c}\text { Node } 5 \\
\text { input }\end{array}$ \\
\hline Gene weights & $\begin{array}{l}\text { IN } 1 \\
\text { OUT } 4 \\
\text { Weight } 0.4 \\
\text { Enable } \\
\text { Innov } 1\end{array}$ & $\begin{array}{l}\text { IN } 2 \\
\text { OUT } 4 \\
\text { Weight } 0.1 \\
\text { Enable } \\
\text { Innov } 2\end{array}$ & $\begin{array}{l}\text { IN } 3 \\
\text { OUT } 5 \\
\text { Weight } 0.2 \\
\text { Enable } \\
\text { Innov } 3\end{array}$ & $\begin{array}{l}\text { IN } 4 \\
\text { OUT } 5 \\
\text { Weight } 0.4 \\
\text { Disable } \\
\text { Innov } 4\end{array}$ & $\begin{array}{l}\text { IN } 3 \\
\text { OUT } 5 \\
\text { Weight } 0.2 \\
\text { Enable } \\
\text { Innov } 5\end{array}$ \\
\hline
\end{tabular}

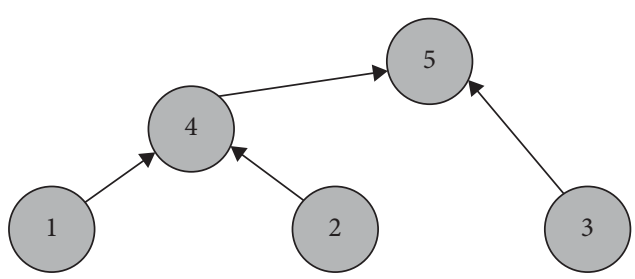

FIgURE 3: NEAT's coding diagram.

inheriting genes with the same ID, and they must represent the same structure. When NEAT mutates, such as if it has a structural mutation, a new and unique innovation ID is assigned to it. For example, as shown in Figure 4, when a new connection 3 through 5 is added, an innovation ID7 is assigned to the connection, and when a node 6 is added, two innovation ids 8 and 9 are assigned to the new connection. Here the innovation ID is global and will be kept throughout the training of the algorithm. This is one of the reasons why NEAT has a strong learning ability.

The variation operation of NEAT can change the topology of the network and the weight in the network, which can be divided into four operations: adding a node, increasing the connection between nodes, adjusting the weight, and changing the excitation function and numerical response. As shown in Figure 4, when the connection between nodes is added, the innovation ID can be directly added to the gene and assigned. When adding a node, the previous connection is disconnected and set to "Disable" before two gene points are added, the new connection method is recorded, and the innovation ID is assigned.

In contrast, NEAT crossover operation is more complex and requires the assistance of innovation ID. In the first two parent genes, all gene points are arranged in ascending order of innovation ID, and then the crossover operation often produces three situations. When two parent gene points are identical, the gene point is called matching gene point. If one of the two parent classes has the innovation ID, and the other does not contain the innovation ID, the gene point is called disjointed gene point. When all gene points of one parent gene are processed, all the remaining gene points of the other parent gene are called excess gene. The rules for the generation of neutrons in crossover operation are as follows. If it is a matching gene point, the gene point is directly used in the daughter gene. If it is a disjointed gene point or an excess gene, it will be obtained from the father with high fitness, and if the two fathers have the same fitness, it will be inherited randomly. In addition, when there are two 


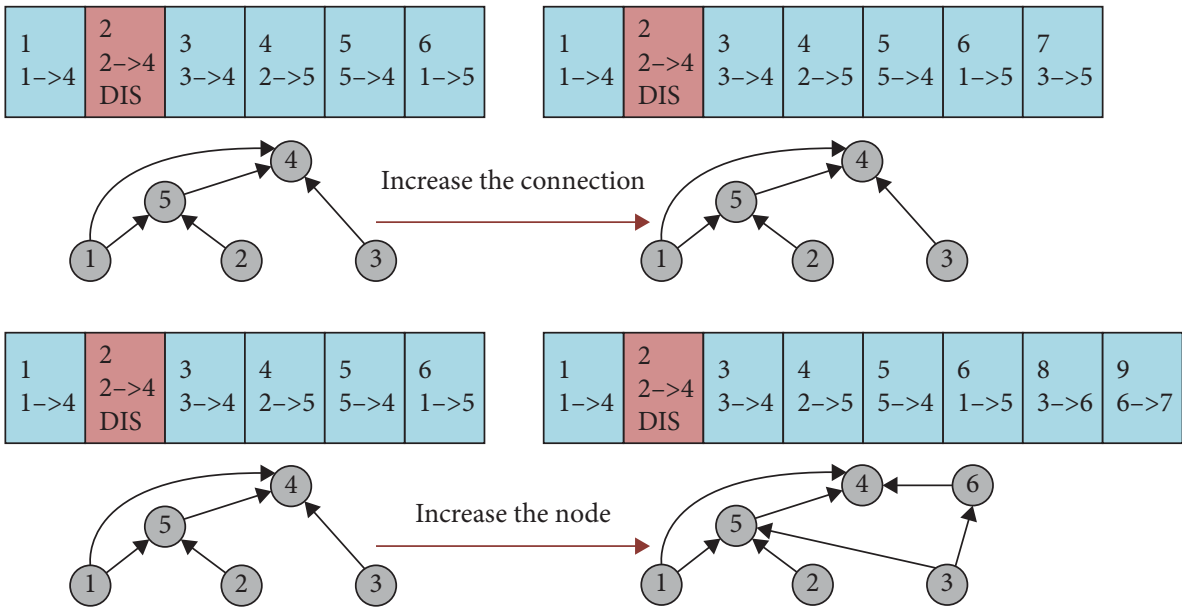

Figure 4: Two types of NEAT variation.

matched gene points, one is Disabled and the other is Enabled, the inherited gene point is Disable.

The last part is the generation and conservation of new populations. Firstly, NEAT's definition of population classification is different from traditional evolutionary algorithms. NEAT has a well-defined Compatibility Function, as shown in formula (1), where $\delta$ represents the difference distance, $E$ represents the number of excess gene points in two genes, $D$ represents the number of disjointed gene points in two genes, $C$ is the coefficient, $W$ is the average weight difference of matched gene points, and $N$ is the length of the longer gene in two genes.

$$
\delta=\frac{c_{1} E}{N}+\frac{c_{2} D}{N}+c_{3} * \bar{W} .
$$

Then, having sorted the species into groups based on the distance between their genes, there is the question of how to select paternal genes for reproduction. NEAT defines the explicit fitness method here, in which the genes of the species belonging to the same group share their environmental fitness. In this way, the high adaptability of some genes to the environment in a group will cover up the other genes with low fitness and reduce the diversity of the whole species. The explicit fitness is shown in formula (2).

$$
f_{i}^{\prime}=\frac{f_{i}}{\sum_{j=1}^{n} \operatorname{sh}(\delta(i, j))} .
$$

Through such fitness sharing, NEAT can constrain each gene. If there are too many members in a group, its fitness evaluation will be reduced. And when new genes are produced, they give protection. Such old genes would be phased out if, over many generations, their environmental fitness had not improved. Figure 5 shows the running process of convolutional neural network based on NEAT algorithm.

3.2. Algorithm Improvement. NEAT algorithm is an improvement on the original topology and weight evolutionary neural network algorithm. Its main improvements can be summarized as follows. First, a more efficient coding scheme is defined. NEAT algorithm sets up two genes to preserve the structural parameters and weights of neural network, which can greatly facilitate crossover operation and mutation operation. Not only did it allow a population to evolve a richer set of genes over time, it also ensures the efficiency of the runtime. Second, set up groups and new species protection mechanisms. Through the definition of differential distance, NEAT algorithm groups new genes according to the differential distance and implements intragroup shared fitness, so that species will not be eliminated early when they are born, ensuring that each species has the opportunity to fully evolve. Finally, at the beginning of NEAT algorithm, a network without any hidden nodes will be generated, which can be called the initial network. With the evolution from generation to generation, the network grows continuously by adding nodes or increasing node connections and selects the reproduction of the network by fitness and eliminates the network with poor fitness. Because the initial network of NEAT is the smallest structure of the network, NEAT algorithm can always search for a lower dimensional space than algorithms such as the fixed topology evolutionary neural network. Compared with other similar evolutionary neural network algorithms, NEAT algorithm constructs a simpler network structure and has advantages in performance.

However, NEAT algorithm has made many improvements on the original TWEANN algorithm, greatly improving the algorithm effect, but there are still some problems in the weight variation part. In the selection of the weight variation algorithm for NEAT algorithm, the original TWEANN weight variation algorithm is still used, that is, Gaussian variation, also known as Gaussian perturbation algorithm, as shown in formula (3).

$$
w_{i}^{\prime}=w_{i}+M_{-} \text {PowerN }(0,1),
$$

where $N(0,1)$ is a random number sampled from random variables subject to normal distribution, $w_{i}$ is the weight of variable and different operations, and $M_{-}$is the value set artificially during training, which can control the size of variation value. There are two problems in this variation of 


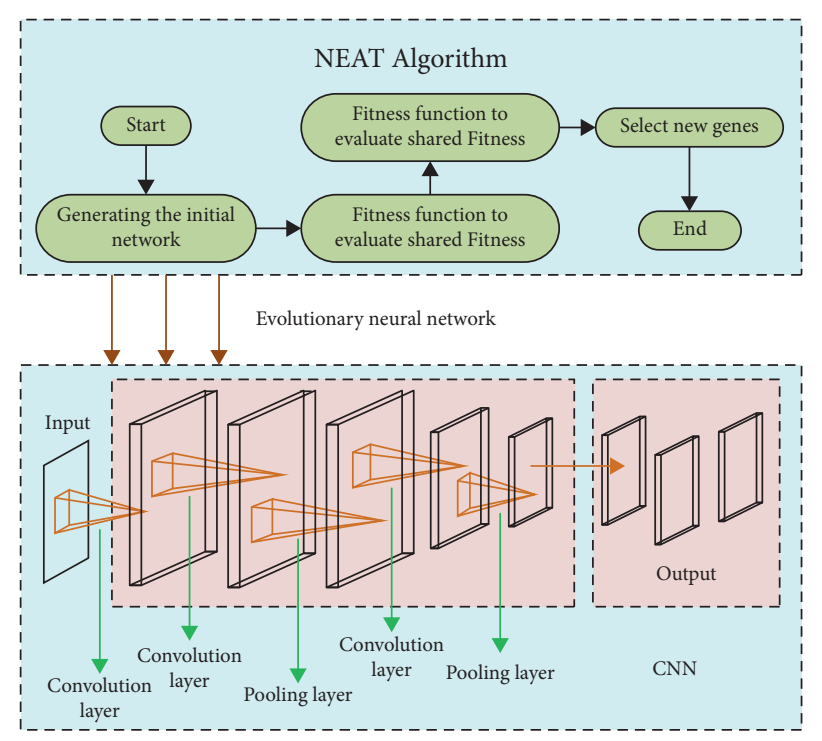

Figure 5: Two types of NEAT variation.

weight value: (1) Because it is a random number sampled from a random variable subject to normal distribution, the variation direction of its weight cannot be controlled, and it is likely to be opposite to the required variation direction in practical application. (2) The perturbation value is a random number sampled from the random variable subject to normal distribution, but its value size still cannot be accurately determined. In practical application, it is likely to produce weight variation far larger than or far smaller than the need, both of which will affect the efficiency of the algorithm.

In practice, in the vast majority of cases in the training model of gradient information can be obtained, while there are a lot of gradient information for incomplete or inaccurate gradient information, etc.; these conditions may result in the gradient descent method training the neural network slow training speed; the training effect is poor and cannot even train problems. However, when gauss variation is used in evolutionary algorithm to change weight, gradient information is not needed, but the direction and value of mutation operation are difficult to determine. This section envisages using gradient information assisted Gaussian variation algorithm to solve two problems in weight variation. Firstly, we need to define disturbance $\delta$. In the Gaussian mutation algorithm before the change, the disturbance is the random number sampled from the random variable subject to normal distribution; that is, the $\delta$ can control the change of the value. So the weight variation algorithm can be rewritten as formula (4).

$$
w^{\prime}=w+\delta M_{\text {Power }}
$$

Secondly, the disturbance $\delta$ should be decomposed into the product of two values, one of which is the change $\delta_{\text {magnitude }}$ and the other is the change $\delta_{\text {direction }}$, as shown in formula (5). This is where gradient information can be used, because in gradient descent, in order to minimize the error function, you need to constantly adjust the weights, and the direction of adjustment is gradient phase. When the weight is disturbed, this $E$ can also be used to identify and combine information in the network; that is, the $\delta_{\text {direction }}$ is also set to the opposite direction of the gradient, so as to solve the problem that the direction of weight variation is opposite to the direction needed in the practical application.

$$
\delta=\delta_{\text {direction }} \delta_{\text {magnitude }} .
$$

Then, the $\delta_{\text {magnitude }}$ change should be determined, and the gradient information should still be used. The steps are as follows:

(1) Assume that the gradient at weight $w_{i}$ is $\Delta w_{i}$, and $r$ is weight $w_{i}$. So the cumulative gradient at the weight can be calculated:

$$
r=r+\left(\Delta w_{i}\right)^{2}
$$

(2) To calculate the update equation formula (7), $\operatorname{abs}(N(0,1))$ is a random number sampled from random variables subject to normal distribution, but only its size is considered here. This random number is divided by $\sqrt{r}$ to ensure that the variation range of the control value is not too large or too small. $\varepsilon$ is a small constant in case the denominator is 0 .

$$
\delta_{\text {magnitude }}=\frac{\operatorname{abs}(N(0,1))}{\varepsilon+\sqrt{r}} .
$$

Finally, the new weight variation algorithm can be written into

$$
w^{\prime}=w+\delta_{\text {direction }} M_{-} \text {Power } \frac{\operatorname{abs}(N(0,1))}{\varepsilon+\sqrt{r}} .
$$

The new weight variation algorithm uses gradient information to assist Gaussian variation algorithm, which provides direction information and limits the range of weight variation reasonably in the process of weight variation, which can further improve the efficiency of neural 
network evolution in training. It should be noted that the new weight variation algorithm does not completely depend on the gradient information. When the gradient information is incomplete or inaccurate, it can still be used as auxiliary information of Gaussian variation algorithm to help carry out more effective weight variation. However, in rare cases where gradient information cannot be obtained completely, the weight variation algorithm will degenerate into the original Gaussian variation algorithm, which will not affect the training of NEAT algorithm itself.

\section{Experiment and Results}

In order to test the effectiveness of promotion recommendation optimization model, we analyze the improvement effect of promotion recommendation optimization model on promotion revenue by using electronic product data of Amazon website. Firstly, the proposed model is compared with the benefits of the two traditional strategies to verify the effectiveness of the model. Secondly, the proposed algorithm is compared with the solution results of tabu search, simulated annealing, and other intelligent search methods to verify the effectiveness of the proposed model solution method.

4.1. Experimental Data Source. On December 17, 2010, Amazon launched a price promotion for the Canon SD13001S camera and offered recommendations. We collect raw prices, promotional discounts, and recommended products (SUB, COM, and IND) from Amazon's promotional practices. Market research [25] and transaction data analysis [26] are effective methods to obtain consumer reserve prices. However, this paper carries out experimental design based on the marketing practice of Amazon.com. On the one hand, it is difficult for us to obtain transaction records of Amazon.com; on the other hand, the large number of products makes it difficult to obtain consumers' reserve prices through market research. Price is the result of continuous improvement in the fierce market competition environment. Therefore, this paper assumes that the selling price of products on Amazon is the optimal price when each product is sold separately.

4.2. The Experimental Results. Firstly, we examine the benefits of enterprises adopting the promotion recommendation optimization model and compare them with the following strategies: (1) PNR strategy: It is assumed that enterprises promote with the optimal price discount obtained in this paper but do not recommend related products; (2) MCR strategy: Attracting consumers to buy products with the highest profit margin is a marketing practice widely adopted by enterprises. MCR strategy assumes that the enterprise promotes with the optimal price discount obtained in this paper and recommends the 10 products with the lowest cost ratio (cost/sales price) from SUB, COM, and ND. Table 1 shows the average promotion demand and revenue of different promotion strategies.
As it can be seen from Table 2, with regard to PNR strategy, enterprises give up the opportunity to guide consumers' purchasing decisions with personalized recommendation and thus lose the possibility to improve promotion income with personalized recommendation. On the other hand, companies cannot use personalized product recommendations to show consumers alternatives to promotional products. Since alternatives generally have higher profit margins than promotional products, the inability to attract consumers to buy alternatives has a negative impact on improving promotional earnings. Therefore, although PNR strategy can sell more promotional products, it gets the worst promotional income. Compared with PNR and MCR strategies; the promotion recommendation optimization model can significantly improve the promotion effect.

To verify the effectiveness of the evolutionary neural network, the results were compared with those of simulated annealing and tabu search. In order to compare the solution effects of the three methods, this chapter takes the data of "Canon SD13001S" camera as an example to conduct 10 simulation experiments. In each experiment, we simulated different consumer retention price, product cost, complementarity coefficient, and dynamic parameters and then solved the model using three methods, respectively, as shown in Figures 6 and 7. It can be seen that evolutionary neural network has more extensive application in practice, so this paper chooses evolutionary neural network algorithm as the solution method of promotion recommendation optimization model.

The interdependence between promotional price and product recommendation determines that enterprises must systematically consider the price discount and recommended product set of promotional products. Given the recommended product set, there is an optimal price discount that can bring the maximum promotion income for enterprises. When the price discount exceeds the optimal discount, further price reduction cannot generate more promotion income. In order to understand the necessity of recommending the right product, we changed the recommended product mix of the enterprise based on the optimal price discount. Figure 8 shows the suboptimal returns for recommended products.

As it can be seen from Figure 8, when the promotional products are sold at the optimal price discount, the enterprise cannot obtain the maximum promotional benefits without recommending the optimal product mix. Therefore, we get the following enlightenment: given the optimal price discount of promotional products, enterprises should recommend reasonable product sets to obtain the optimal promotional income.

This section changes the product cost, retention price, complementarity coefficient, and other parameters within a reasonable range, verifies the stability of the promotion recommendation optimization model in the face of uncertain environment, and provides more general suggestions for the promotion practice of enterprises. This section firstly verifies the influence of product cost on promotion revenue and promotion demand, then verifies the influence of consumer reserve price type and range, and finally verifies 
TABle 1: Performance comparison between our model and other strategies.

\begin{tabular}{|c|c|c|c|c|}
\hline \multicolumn{2}{|c|}{ Sales promotion strategy } & PRN & MCR & Our model \\
\hline \multirow{4}{*}{ Sales income } & Promotional products & -1980 & -1883 & -1952 \\
\hline & Substitute & -19312 & -16263 & -17986 \\
\hline & Complements & 24921 & 25635 & 27159 \\
\hline & Independent product & 20233 & 19612 & 23243 \\
\hline \multicolumn{2}{|c|}{ Total promotion revenue } & 23862 & 27101 & 30464 \\
\hline \multirow{4}{*}{ Promotion needs } & Promotional products & 597 & 555 & 586 \\
\hline & Substitute & -594 & -505 & -555 \\
\hline & Complements & 2117 & 2205 & 2310 \\
\hline & Independent product & 1786 & 1781 & 1969 \\
\hline \multicolumn{2}{|c|}{ Total promotion demand } & 3906 & 4036 & 4310 \\
\hline
\end{tabular}

TABLE 2: Impacts of cost ratio.

\begin{tabular}{lccccccc}
\hline \multirow{2}{*}{$\begin{array}{c}* \\
\text { Cost rate }\end{array}$} & Promotional discounts & PWR & MCR & Our model & PWR & Supply requirements $(* 103)$ \\
& & 5.72 & 5.76 & $\mathbf{6 . 1 7}$ & 7.73 & 8.18 \\
OCR & Our model \\
\hline 50 & 51 & 5.13 & 5.23 & $\mathbf{5 . 6 4}$ & 4.62 & 5.26 & $\mathbf{9 . 2 0}$ \\
70 & 57 & 3.98 & 4.11 & $\mathbf{4 . 4 3}$ & 2.49 & 3.04 & $\mathbf{5 . 9 5}$ \\
80 & 68 & 2.28 & 2.39 & $\mathbf{2 . 6 0}$ & 0.92 & 1.29 & $\mathbf{3 . 4 9}$ \\
90 & 81 & 0.71 & 0.84 & $\mathbf{0 . 8 7}$ & 0.10 & 0.34 \\
\hline
\end{tabular}

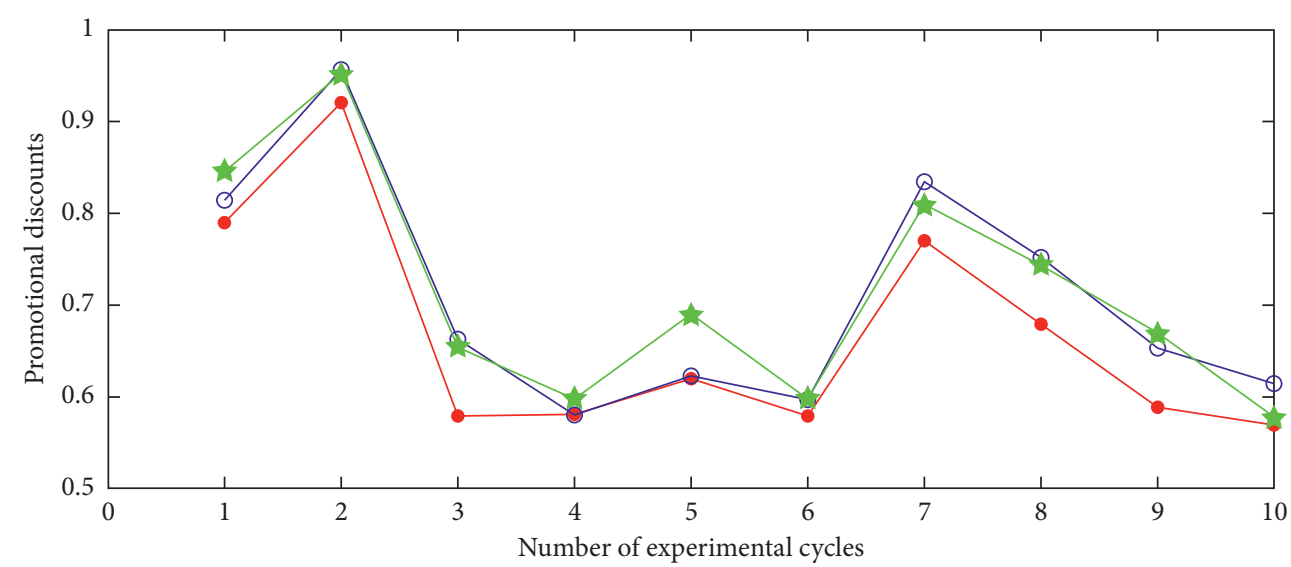

- Our model

- Simulated annealing

th Tabu search

FIGURE 6: Experimental comparison of promotional discounts.

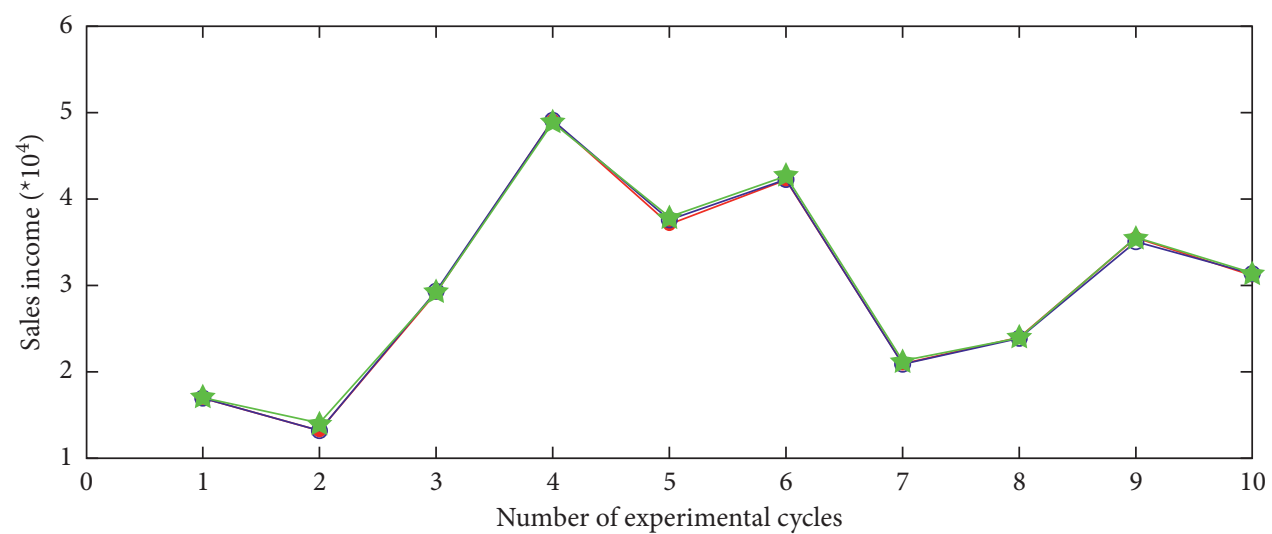

- Our model

- Simulated annealing

th Tabu search

Figure 7: Experimental comparison of sales income. 


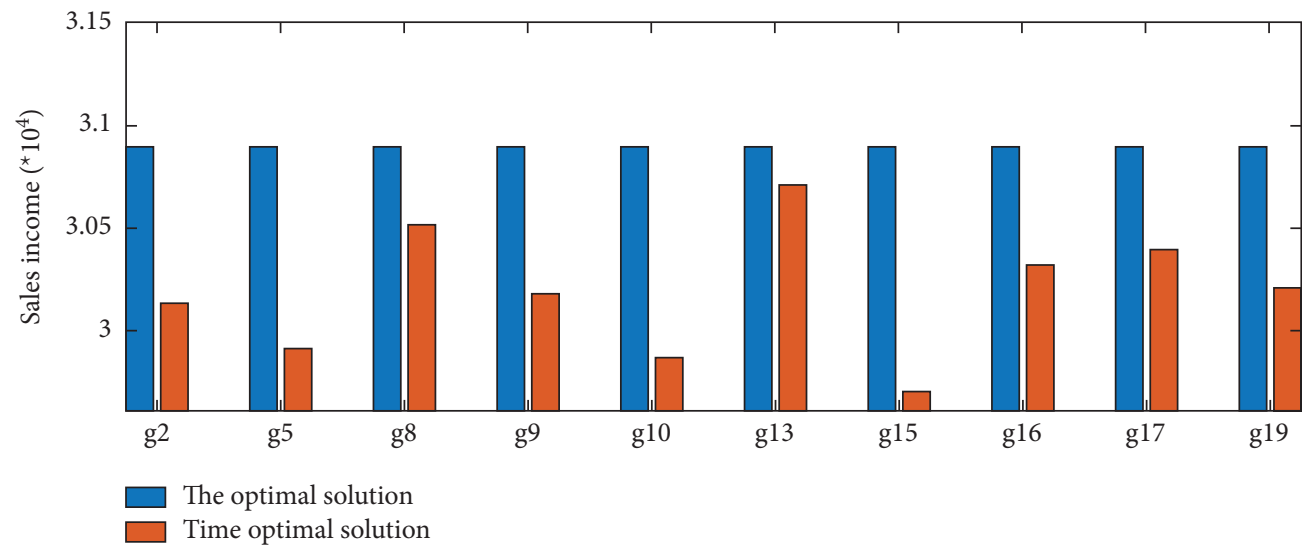

FIGURE 8: Experimental comparison of sales income.

the influence of complementarity coefficient, dynamic parameters, and consumer purchase decision uncertainty on the model effect. The data source used in the experiment is from the promotion practice of 50 electronic products on Amazon website on December 19, 2010.

As it can be seen from Table 2, promotion demand and promotion revenue decrease with the increase of cost rate. For promotional products with high cost rate, large price discounts are easy to cause serious losses, and it is difficult to attract consumers' attention if attractive price discounts are not provided. For other products, higher cost rates on the one hand push up product prices. On the other hand, they reduce the ability of enterprises to profit from recommended products. When the product cost rate increases from $50 \%$ to $90 \%$, the average price discount increases from $51 \%$ to $93 \%$, while the promotion demand and promotion revenue decrease from 9200 and 61700 to 420 and 8700 , respectively. This shows that product cost rate is the key factor affecting the promotion revenue.

\section{Conclusion}

Under the environment of electronic commerce, the demand of consumers is becoming more and more diversified, and the difference is also becoming more and more significant. The fierce market competition makes the modeling of consumers' personalized needs and the formulation of corresponding strategies to guide consumers' purchase decisions become a hot and difficult research topic in the field of e-commerce, which is also an important topic related to the survival and development of enterprises. This article designed a method based on evolutionary neural network model: in order to achieve the maximum profit of sales promotion, this paper put forward the use of discounts to attract consumers; the use of recommended products make up the loss to the promotional products promotion and recommendation strategy. Promotion recommendation optimization model can help enterprises make decisions in the following two aspects: (1) what is the optimal discount of promotional products? (2) which products can be recommended to maximize promotional revenue? The relationship between promotional discounts and product recommendations as well as the characteristics of promotional products and recommended products are deeply analyzed by using the product data of Amazon website.

How to convert potential consumers into purchasers is a widespread concern of enterprises, and it is also a difficult problem in the field of pricing research to make personalized prices according to consumers' purchasing decision-making process. This paper combines personalized recommendation with bundled pricing strategy, which provides a brand new research idea for personalized recommendation field and pricing strategy considering real-time characteristics of network environment. Experimental results show that the evolutionary neural network model can provide attractive personalized price for consumption. The results of this paper extend the research ideas of personalized marketing strategy, enrich the method system of modeling and solving personalized marketing strategy, and provide a more systematic solution for the modeling and optimization of personalized marketing strategy.

\section{Data Availability}

The datasets used during the current study are available from the corresponding author on reasonable request.

\section{Conflicts of Interest}

The author declares that he has no conflicts of interest.

\section{References}

[1] J. Deng, "Research on the construction and optimization strategy of marketing based on big data era," China Market, vol. 32, pp. 118-119, 2021.

[2] S. Moorthy and M. Ding, "On marketing strategy in electoral politics," Customer Needs and Solutions, vol. 6, no. 3-4, pp. 57-62, 2019.

[3] V. Shankar, A. K. Smith, and A. Rangaswamy, "Customer satisfaction and loyalty in online and offline environments," International Journal of Research in Marketing, vol. 20, no. 2, pp. 153-175, 2003.

[4] V. Souitaris and G. Balabanis, "Tailoring online retail strategies to increase customer satisfaction and loyalty," Long Range Planning, vol. 40, no. 2, pp. 244-261, 2007. 
[5] L. Ji, S. Yang, and L. Li, "Marketing strategy analysis of chengde lulu based on $4 \mathrm{P}$ marketing theory," China Collective Economics, vol. 34, pp. 69-70, 2021.

[6] Z. Chen and L. Wang, "Personalized product configuration rules with dual formulations: a method to proactively leverage mass confusion," Expert Systems with Applications, vol. 37, no. 1, pp. 383-392, 2010.

[7] S. Schneider, P. Shabalin, and M. Bichler, "On the robustness of non-linear personalized price combinatorial auctions," European Journal of Operational Research, vol. 206, no. 1, pp. 248-259, 2010.

[8] A. L. Montgomery and M. D. Smith, "Prospects for personalization on the internet," Journal of Interactive Marketing, vol. 23, no. 2, pp. 130-137, 2009.

[9] T. Levina, Y. Levin, J. McGill, and M. Nediak, "Dynamic pricing with online learning and strategic consumers: an application of the aggregating algorithm," Operations Research, vol. 57, no. 2, pp. 327-341, 2009.

[10] H. Y. Wang, R. Wang, and M. D. Wang, "Analysis of leading factors of marketing based on intelligent BP neural network," New Generation information Technology, vol. 3, no. 9, pp. $28-32,2020$.

[11] D. F. Specht, "Probabilistic neural networks," Neural Networks, vol. 3, no. 1, pp. 109-118, 1990.

[12] A. Talukder and D. Casasent, "A closed-form neural network for discriminatory feature extraction from high-dimensional data," Neural Networks, vol. 14, no. 9, pp. 1201-1218, 2001.

[13] A. Vellido, P. J. G. Lisboa, and K. Meehan, "Segmentation of the on-line shopping market using neural networks," Expert Systems with Applications, vol. 17, no. 4, pp. 303-314, 1999.

[14] C. C. Chang, P.-L. Chen, F.-R. Chiu, and Y.-K. Chen, "Application of neural networks and Kano's method to content recommendation in web personalization," Expert Systems with Applications, vol. 36, no. 3, pp. 5310-5316, 2009.

[15] T. Bäck and H.-P. Schwefel, "An overview of evolutionary algorithms for parameter optimization," Evolutionary Computation, vol. 1, no. 1, pp. 1-23, 1993.

[16] L. D. Whitley and T. Hanson, "Optimizing neural networks using FasterMore accurate genetic search," in Proceedings of the 3rd International Conference on Genetic Algorithms, pp. 391-397, San Francisco, CA, USA, 1989.

[17] S. Lander and Y. Shang, "EvoAE - a new evolutionary method for training autoencoders for deep learning networks," in Proceedings of the 2015 IEEE 39th Annual Computer Software and Applications Conference, pp. 790-795, Taichung, China, 2015.

[18] K. W. C. Ku, M. W. Man Wai Mak, and W. C. Wan-Chi Siu, "A study of the Lamarckian evolution of recurrent neural networks," IEEE Transactions on Evolutionary Computation, vol. 4, no. 1, pp. 31-42, 2000.

[19] J. Lehman, J. Chen, and J. Clune, "Safe mutations for deep and recurrent neural networks through output gradients," in Proceedings of the Genetic and Evolutionary Computation Conference, pp. 117-124, Kyoto, Japan, 2018.

[20] E. Real, S. Moore, and A. Selle, "Large-scale evolution of image classifiers," in Proceedings of the 34th International Conference on Machine Learning, vol. 70, pp. 2902-2911, Sydney, Australia, 2017.

[21] M. S. C. Rusuli, R. Ruwaida, M. Noraani, J. Takala, and M. Nizamuddin, "Influence of marketing mix strategy in insurance business: the case of Kota Bharu," Research in World Economy, vol. 10, no. 2, p. 88, 2019.
[22] A. Rap and B. Vlc, "Exploring customer orientation as a marketing strategy of Mexican-American entrepreneurs," Journal of Business Research, vol. 113, pp. 139-148, 2020.

[23] K. K. Halim, S. Halim, and Felecia, "Business intelligence for designing restaurant marketing strategy: a case study," Procedia Computer Science, vol. 161, pp. 615-622, 2019.

[24] S. Yao and C. F. Mela, "Online auction demand," Marketing Science, vol. 27, no. 5, pp. 861-885, 2008.

[25] N. A. Morgan, K. A. Whitler, H. Feng, and S. Chari, "Research in marketing strategy," Journal of the Academy of Marketing Science, vol. 47, no. 1, pp. 4-29, 2018.

[26] C. D’Souza, T. Marjoribanks, and S. Young, "Environmental management systems: an alternative marketing strategy for sustainability," Journal of Strategic Marketing, vol. 27, no. 5, pp. 417-434, 2019. 